\title{
Simple Reduction of Gamma-Ray Bursts
}

\author{
Xiang Liu \\ NAOC, South Beijing Road 40-5, Urumqi 830011, China
}

\begin{abstract}
We try to explain the different type of GRBs from their properties, most likely the long duration GRBs are the NS-NS collision, short duration ones are the WD-WD collision, and intermediate ones are the NS-WD collision.
\end{abstract}

\section{Hypernova/supernova}

For Gamma-Ray Burst (GRB), its total energy is about the same as a supernova assuming that all of the energy comes out in gamma-rays. If there is inefficiency in conversion of energy to gamma-rays, the total energy must be even greater. However, GRB afterglows are so faint that they are not much greater than the limit of detection devices. Supernovae are easier to find because they're generally much brighter than GRB afterglows. GRBs could originate in a different way compared to normal supernova. A few GRBs are probably associated with supernovae, e.g. SN1998bw, however, it is a unusual one could be produced from NS-NS collision rather than a single star explosion. And collision can easily produce strong beaming jet which supposed to be common in GRBs.

\section{Accretion in binary system}

For close binary systems, e.g. X-ray binaries, there is accretion which leads to emission or an explosion. A very young neutron star and/or neutron star with some material falling onto it could create the X-ray burster and/or the soft gamma-ray repeater (hard X-ray flasher). Magnetar could be due to accretion and/or be very young neutron star with an active dynamo.

\section{Collision of 2 compact objects}

\subsection{Neutron star-neutron star collision}

The GRBs with longer duration of $>2$ seconds, they have total energy 20 times of those GRBs with duration of $<2$ seconds (Mao et al. 1994), should originate in a strong collision. Neutron star-neutron star collision is the first possibility to be responsible for this GRB subgroup. 


\subsection{White dwarf-white dwarf collision}

The GRBs with short duration of $<2$ seconds, they have total energy of 20 times less than that of long duration GRBs, should originate in a less strong collision. White dwarf-white dwarf collision could be account for the short duration GRBs.

\subsection{Neutron star-white dwarf collision}

It was suggested that there is the third class of GRBs which have intermediate duration and intermediate fluence (Hakkila et al. 2000). Neutron star-white dwarf collision might be responsible for the intermediate GRB group.

\section{References}

Mao, S., Narayan, R., \& Piran, T. 1994, ApJ, 420, 171

Hakkila, J., Haglin, D.J., Pendleton, G.N., et al. 2000, ApJ, 538, 165 